\title{
OPERATORS IN THE COMMUTANT OF A REDUCTIVE ALGEBRA
}

\author{
ROBERT L. MOORE
}

\begin{abstract}
Let $\mathcal{Q}$ be a reductive algebra. It is shown that there is a subspace $\mathscr{R}$ that reduces $\mathbb{Q}$ and such that the commutant of $\mathbb{Q} \mid \mathfrak{T}$ is selfadjoint and the commutant of $Q \mid \Re^{\perp}$ consists of hyporeductive operators. It is then shown that under a variety of conditions, if an operator $T$ is in $\mathbb{Q}^{\prime}$, then $T^{*}$ is in $\mathbb{Q}^{\prime}$.
\end{abstract}

In [5], we made use of a decomposition of reductive operators on Hilbert space to deduce some results concerning the selfadjointness of the commutants of such operators. In that paper we also observed that a similar decomposition of a reductive operator algebra is possible. In this paper we produce such a decomposition and use it to answer a number of questions first raised by Rosenthal [9].

By an algebra, we will mean a weakly closed subalgebra (with identity) of the algebra of all (bounded) operators on a separable Hilbert space. If $\mathbb{Q}$ is an algebra and $\mathfrak{N}$ a subspace of $\mathcal{H}$ then $\mathbb{Q} \mathfrak{N}=\{A f: A \in \mathbb{Q}$ and $f \in \mathscr{N}\}$, and $\mathscr{N}$ is invariant for $\mathbb{Q}$ if $\mathscr{Q} \mathscr{T} \subseteq \Re$. The lattice of subspaces invariant for $\mathbb{Q}$ is denoted by Lat $\mathcal{Q}$. We also denote by $\mathcal{Q}^{*}$ the algebra $\left\{A^{*}: A \in \mathbb{Q}\right\}$, and by $\mathcal{Q}^{\prime}$ the algebra $\{B: A B=B A$ for all $A \in \mathbb{Q}\}$. Finally, $Q$ is reductive if $\mathfrak{N} \in$ Lat $\mathbb{Q}$ implies $\mathfrak{M}^{\perp} \in$ Lat $\mathbb{Q}$, or equivalently, if Lat $\mathbb{Q}=$ Lat $\mathbb{Q}^{*}$.

The results for which we are aiming are Theorems 2 and 3, which state that for every reductive algebra $\mathbb{Q}$ there is a subspace $\mathfrak{T}_{0} \in$ Lat $\mathbb{Q} \cap$ Lat $\mathbb{Q}^{\prime}$, such that $\mathbb{Q}^{\prime} \mid \Re_{0}$ is selfadjoint and $\operatorname{Lat}\left(\mathscr{Q} \mid \mathfrak{T}_{0}^{+}\right) \subseteq \operatorname{Lat}\left(\mathbb{Q}^{\prime} \mid \mathfrak{T}_{0}^{+}\right)$. Before we begin, we remark that certain techniques developed by Hoover [4] will also yield Theorems 2 and 3.

THeOREM 1. Let $\mathbb{Q}$ be a reductive algebra and $\Re \in$ Lat $\mathbb{Q}$. Let $X: \Re^{\perp} \rightarrow$ $\mathfrak{N}$ and suppose that $T \in \mathbb{Q}^{\prime}$, where $T$ has the form

$$
T=\left(\begin{array}{cc}
0 & X \\
0 & 0
\end{array}\right)
$$

according to the decomposition $\mathscr{H}=\mathscr{M} \oplus \mathfrak{K}^{\perp}$. Then

(1) $T^{*} \in \mathbb{Q}^{\prime}$.

(2) $(\operatorname{ran} T)^{-} \in$ Lat $\mathbb{Q}$ and $\left(\mathbb{Q} \mid(\operatorname{ran} T)^{-}\right)^{\prime}$ is selfadjoint.

(3) $\operatorname{ker} T \in$ Lat $Q$ and $\left(Q \mid \operatorname{ker}^{\perp} T\right)^{\prime}$ is selfadjoint.

Received by the editors February 8, 1977.

AMS (MOS) subject classifications (1970). Primary 47C05; Secondary 47D99.

Key words and phrases. Reductive algebra, hyperinvariant subspace, hyporeductive operator. 
Proof. (1) All decompositions of vectors and operators are with respect to $\Re$ and $\Re^{\perp}$. Let $\Re$ be the subspace $\left\{\langle X f, f\rangle: f \in \Re^{\perp}\right\}$. Since $T \in \mathbb{Q}^{\prime}$ it is easy to check that $\Re \in$ Lat $Q$, and thus the subspace $\Re^{\perp}=\{\langle g$, $\left.\left.-X^{*} g\right\rangle: g \in \mathfrak{N}\right\}$ also lies in Lat $\mathbb{Q}$.

If $A \in \mathbb{Q}$ we can decompose $A$ as $A_{1} \oplus A_{2}$, since $\mathcal{Q}$ is reductive. If $g \in \Re$ we have

$$
\left(A_{1} \oplus A_{2}\right)\left\langle g,-X^{*} g\right\rangle=\left\langle A_{1} g,-A_{2} X^{*} g\right\rangle .
$$

Since this vector must lie in $\mathcal{T}^{\perp}$ it follows that $-X^{*} A_{1} g=-A_{2} X^{*} g$ for any $g \in \mathfrak{R}$, and hence that $X^{*} A_{1}=A_{2} X^{*}$, which implies that $T^{*} \in \mathbb{Q}^{\prime}$.

(2) Since $T \in \mathbb{Q}^{\prime},(\operatorname{ran} T)^{-} \in$ Lat $Q$. Let $C$ be an operator on $(\operatorname{ran} T)^{-}$ that lies in the commutant of $Q \mid(\operatorname{ran} T)^{-}$; we want to show that $C^{*} \in$ $\left(\mathscr{Q} \mid(\operatorname{ran} T)^{-}\right)^{\prime}$. Let $C_{1}$ be the operator on $\mathcal{H}$ defined by

$$
C_{1} f= \begin{cases}C f, & f \in(\operatorname{ran} T)^{-}, \\ 0, & f \in \operatorname{ran}^{\perp} T .\end{cases}
$$

Then $C_{1} \in \mathbb{Q}^{\prime}$ and it will suffice to show that $C_{1}^{*} \in \mathbb{Q}^{\prime}$.

Let $T_{1}=C_{1} T$; then $T_{1} \in \mathbb{Q}^{\prime}$. Moreover, since $\mathscr{T}$ is invariant under $C_{1}$, it is easy to see that $T_{1}$ has the same form as $T$, namely

$$
T_{1}=\left(\begin{array}{ll}
0 & * \\
0 & 0
\end{array}\right) \text {. }
$$

It follows by part (1) that $T_{1}^{*} \in \mathbb{Q}^{\prime}$, that is, for any $A \in \mathbb{Q}, T^{*} C_{1}^{*} A=$ $A T^{*} C_{1}^{*}$. Since $T^{*} \in \mathbb{Q}^{\prime}, T^{*}\left(A C_{1}^{*}-C_{1}^{*} A\right)=0$, that is, $\operatorname{ran}\left(A C_{1}^{*}-C_{1}^{*} A\right) \subseteq$ ker $T^{*}$. On the other hand $\operatorname{ran} C_{1}^{*} \subseteq(\operatorname{ran} T)^{-}$and $(\operatorname{ran} T)^{-}$reduces $A$, so that

$$
\operatorname{ran}\left(A C_{1}^{*}-C_{1}^{*} A\right) \subseteq(\operatorname{ran} T)^{-}=\operatorname{ker}^{\perp} T^{*} .
$$

We conclude that $A C_{1}^{*}-C_{1}^{*} A=0$, and $C_{1}^{*} \in \mathbb{Q}^{\prime}$.

(3) Let $U$ be the unitary operator $\left(\begin{array}{ll}0 & 1 \\ 1 & 0\end{array}\right)$. Since $T^{*} \in \mathbb{Q}^{\prime}$, we have

$$
\left(\begin{array}{cc}
0 & X^{*} \\
0 & 0
\end{array}\right)=U^{*} T^{*} U \in\left(U^{*} \mathscr{U} U\right)^{\prime}
$$

By part (2), $\left[\left(U^{*} \mathscr{Q} U\right) \mid\left(\operatorname{ran} U^{*} T^{*} U\right)^{-}\right]^{\prime}$ is selfadjoint. Thus $\left[Q \mid\left(\operatorname{ran} T^{*}\right)^{-}\right]^{\prime}$ is selfadjoint and (3) follows because (ran $\left.T^{*}\right)^{-}=\operatorname{ker}^{\perp} T$.

Corollary. Let $\mathbb{Q}$ be reductive and $\mathfrak{N} \in$ Lat $\mathcal{Q}$. Let $Y: \mathfrak{K} \rightarrow \mathfrak{K}^{\perp}$ and suppose that $S \in \mathbb{Q}^{\prime}$, where $S=\left(\begin{array}{c}0 \\ Y\end{array} 0\right)$. Then

(1) $S^{*} \in \mathbb{Q}^{\prime}$.

(2) ker $S \in$ Lat $\mathbb{Q}$ and $\left(\mathbb{Q} \mid \operatorname{ker}^{\perp} S\right)^{\prime}$ is selfadjoint.

(3) $(\operatorname{ran} S)^{-} \in$ Lat $Q$ and $(\mathbb{Q} \mid \operatorname{ran} S)^{\prime}$ is selfadjoint.

Proof. Consider adjoints and apply the theorem.

ThEOREM 2. Let $\mathbb{Q}$ be a reductive algebra. There is a subspace $\mathfrak{T}_{0}$ such that

(1) $\mathfrak{M}_{0} \in$ Lat $\mathbb{Q}$;

(2) $\left(\mathbb{Q} \mid \Re_{0}\right)^{\prime}$ is selfadjoint;

(3) there is no nonzero subspace $\Re \subseteq \Re_{0}^{+}$with properties (1) and (2). 
Moreover, this subspace $\mathfrak{T}_{0}$ reduces $\mathbb{Q}^{\prime}$ as well.

Proof. Let $\mathscr{F}=\left\{\mathscr{T} \in\right.$ Lat $\mathbb{Q}:(\mathbb{Q} \mid \mathfrak{T})^{\prime}$ is selfadjoint $\}$. The family $\mathscr{F}$ is nonempty since it contains the zero subspace. Suppose that $\left\{\mathfrak{N}_{\alpha}\right\}_{\alpha \in B}$ is a chain in $\mathscr{F}$; in order to apply Zorn's lemma we would like to show that $\mathscr{N}=\bigvee\left\{\mathscr{T}_{\alpha}: \alpha \in B\right\}$ is also in $\mathscr{F}$, for which it suffices to show that $(\mathbb{Q} \mid \Re)^{\prime}$ is selfadjoint.

Let $T$ be an operator on $\Re$ such that $T \in(\mathscr{Q} \mid \mathfrak{T})^{\prime}$. To show that $T^{*} \in(\mathbb{Q} \mid \Re)^{\prime}$ we must show that for all $A \in \mathbb{Q} \mid \Re$ and for all $f \in \mathscr{R}$ we have $T^{*} A f=A T^{*} f$, and in fact it will be enough to show this equality for all $f \in \cup\left\{\mathfrak{N}_{\alpha}: \alpha \in B\right\}$, because this set is dense in $\mathfrak{N}$. On the other hand, in this case $f \in \mathscr{T}_{\beta}$ for some $\beta \in B$.

Decompose $\mathscr{N}$ as $\mathfrak{K}_{\beta} \oplus\left(\mathfrak{K} \ominus \mathfrak{K}_{\beta}\right)$; then (since $\mathfrak{K}_{\beta} \in$ Lat $\left.\mathscr{Q}\right)$ if $A \in$ Q $\mid \mathfrak{R}$ we have

$$
A=\left(\begin{array}{cc}
A_{1} & 0 \\
0 & A_{2}
\end{array}\right) \text { and } T=\left(\begin{array}{ll}
T_{11} & T_{12} \\
T_{21} & T_{22}
\end{array}\right) .
$$

Because $f \in \mathscr{T}_{\beta}$ we have

$$
T^{*} A f=\left\langle T_{11}^{*} A_{1} f, T_{12}^{*} A_{1} f\right\rangle \quad \text { and } \quad A T^{*} f=\left\langle A_{1} T_{11}^{*} f, A_{2} T_{12}^{*} f\right\rangle .
$$

Since $T_{11} \in\left(\mathscr{Q} \mid \Re_{\beta}\right)^{\prime}$ and $\left(\mathscr{Q} \mid \Re_{\beta}\right)^{\prime}$ is selfadjoint, we have $T_{11}^{*} A_{1}=A_{1} T_{11}^{*}$. Furthermore, the operator $\left(\begin{array}{cc}0 & T_{12} \\ 0 & 0\end{array}\right)$ lies in $(Q \mid \mathcal{T})^{\prime}$ and by Theorem 1 , so does its adjoint $\left({ }_{T_{12}^{*}}^{0}, 0\right)$ and it follows that $A_{2} T_{12}^{*}=T_{12}^{*} A_{1}$. Thus $T^{*} A f=A T^{*} f$, $T^{*} \in(\mathbb{Q} \mid \mathfrak{T})^{\prime}$, and $\mathfrak{R} \in \mathcal{F}$.

By Zorn's lemma there exists a maximal element $\mathfrak{T}_{0}$ of $\mathscr{F} . \mathfrak{T}_{0}$ automatically satisfies requirements (1) and (2) of the theorem. Suppose there is a nonzero subspace $\Re_{1}$ of $\Re_{0}^{\perp}$ for which (1) and (2) hold. We assert that $\mathfrak{K}_{0} \oplus \mathfrak{R}_{1}$ lies in $\mathscr{F}$, a fact which contradicts the maximality of $\mathfrak{K}_{0}$. We must show that $\left(\mathbb{Q} \mid \mathfrak{T}_{0} \oplus \mathfrak{K}_{1}\right)^{\prime}$ is selfadjoint. If $S$ is an operator on $\mathfrak{T}_{0} \oplus$ $\mathfrak{K}_{1}$ such that $S \in\left(\mathbb{Q} \mid \mathscr{T}_{0} \oplus \mathfrak{M}_{1}\right)^{\prime}$ then we decompose $S$ as

$$
\left(\begin{array}{ll}
S_{11} & S_{12} \\
S_{21} & S_{22}
\end{array}\right)
$$

where $S_{11} \in\left(\mathscr{Q} \mid \mathscr{T}_{0}\right)^{\prime}$ and $S_{22} \in\left(\mathbb{Q} \mid \mathscr{T}_{1}\right)^{\prime}$. By assumption $S_{11}^{*} \in\left(\mathbb{Q} \mid \mathscr{T}_{0}\right)^{\prime}$ and $S_{22}^{*} \in\left(\mathbb{Q} \mid \mathscr{T}_{1}\right)^{\prime}$. Moreover, the operator

$$
\left(\begin{array}{cc}
0 & S_{12} \\
0 & 0
\end{array}\right)
$$

satisfies the hypotheses of Theorem 1 for the reductive algebra $\left(Q \mid \mathscr{T R}_{0} \oplus\right.$ $\left.\mathfrak{R}_{1}\right)$. Thus by that theorem the operator

$$
\left(\begin{array}{cc}
0 & 0 \\
S_{12}^{*} & 0
\end{array}\right)
$$

lies in $\left(\mathscr{Q} \mid \mathfrak{T}_{0} \oplus \mathfrak{K}_{1}\right)^{\prime}$. Similarly we use the corollary to Theorem 1 to show that $\left(\begin{array}{cc}0 & s_{21}^{*} \\ 0 & 0\end{array}\right)$ lies in $\left(Q \mid \mathscr{T}_{0} \oplus \mathbb{M}_{1}\right)^{\prime}$. Since 


$$
S^{*}=\left(\begin{array}{cc}
S_{11}^{*} & 0 \\
0 & 0
\end{array}\right)+\left(\begin{array}{cc}
0 & 0 \\
0 & S_{22}^{*}
\end{array}\right)+\left(\begin{array}{cc}
0 & S_{21}^{*} \\
0 & 0
\end{array}\right)+\left(\begin{array}{cc}
0 & 0 \\
S_{12}^{*} & 0
\end{array}\right)
$$

we see that $S^{*} \in\left(\mathbb{Q} \mid \Re_{0} \oplus \mathfrak{K}_{1}\right)^{\prime}$, and thus that $\left(\mathscr{Q} \mid \mathscr{T}_{0} \oplus \mathfrak{K}_{1}\right)^{\prime}$ is selfadjoint.

Finally, to show that $\mathfrak{T}_{0} \in$ Lat $\mathbb{Q}^{\prime}$, suppose that $T \in \mathbb{Q}^{\prime}$ and write

$$
T=\left(\begin{array}{ll}
T_{11} & T_{12} \\
T_{21} & T_{22}
\end{array}\right)
$$

according to the decomposition $\mathscr{H}=\mathscr{T}_{0} \oplus \mathfrak{K}_{0}^{+}$.

Since $\mathfrak{T}_{0}$ reduces $\mathbb{Q}$ the operator

$$
S=\left(\begin{array}{cc}
0 & T_{12} \\
0 & 0
\end{array}\right)
$$

also lies in $\mathbb{Q}^{\prime}$, and by Theorem 1 , ker $S$ reduces $\mathbb{Q}$ and $\left(\mathbb{Q} \mid \operatorname{ker}^{\perp} S\right)^{\prime}$, is selfadjoint. However, $\operatorname{ker}^{\perp} S$ is a subspace of $\mathfrak{T}_{0}^{\perp}$ so by the maximality of $\Re_{0}$ it must be that $\operatorname{ker}^{\perp} S=\{0\}$, that is, $T_{12}=0$. Similarly we can show that $T_{21}=0$ and thus $\mathfrak{M}_{0}$ reduces $T$.

We use the notation Red $\mathscr{B}$ to mean Lat $\mathscr{B} \cap$ Lat $\mathscr{B}^{*}$, where $\mathscr{B}$ is any algebra.

THEOREM 3. Let $\mathbb{Q}$ be reductive and suppose that for no nonzero subspace $\mathfrak{R}$ in Lat $\mathbb{Q}$ is it true that $(\mathbb{Q} \mid \Re)^{\prime}$ is selfadjoint. Then Lat $\mathbb{Q} \subseteq \operatorname{Red} \mathbb{Q}^{\prime}$.

Proof. Suppose $\mathfrak{K} \in$ Lat $\mathbb{Q}$ and $T \in \mathbb{Q}^{\prime}$. Decompose $\mathcal{H}$ as $\mathscr{K} \oplus \mathfrak{K}^{\perp}$ and $T$ as

$$
T=\left(\begin{array}{ll}
T_{11} & T_{12} \\
T_{21} & T_{22}
\end{array}\right)
$$

let

$$
S=\left(\begin{array}{cc}
0 & T_{12} \\
0 & 0
\end{array}\right)
$$

By Theorem 1, $(\operatorname{ran} S)^{-} \in$ Lat $\mathscr{Q}$ and $\left[\mathbb{Q} \mid(\operatorname{ran} S)^{-}\right]^{\prime}$ is selfadjoint. Hence, by the hypothesis of the theorem, $\operatorname{ran} S=\{0\}$, that is, $T_{12}=0$. Similarly $T_{21}=$ 0 and $\mathfrak{R} \in \operatorname{Red} \mathbb{Q}^{\prime}$.

C. K. Fong [1] has used the word hyporeductive to refer to an operator $T$ such that every hyperinvariant subspace of $T$ reduces $T$.

Corollary. Let $\mathbb{Q}$ be as in Theorem 3. If $T \in \mathbb{Q}^{\prime}$ and $\mathfrak{N}$ is hyperinvariant for $T$ then $\mathfrak{N} \in \operatorname{Red} \mathcal{Q}^{\prime}$. In particular, $T$ is hyporeductive.

It follows from all the above that if $\mathscr{Q}$ is any reductive algebra and $T \in \mathbb{Q}^{\prime}$, then $T=T_{1} \oplus T_{2}$ where $T_{1}^{*} \in\left(\mathbb{Q} \mid \mathcal{T}_{0}\right)^{\prime}$ and $T_{2}$ is hyporeductive. Thus if we desire to show that $T^{*} \in \mathbb{Q}^{\prime}$, it suffices to show that $T_{2}^{*} \in\left(\mathbb{Q} \mid T_{0}^{\perp}\right)^{\prime}$.

In [9], P. Rosenthal introduced the following property which an operator $T$ may have in connection with reductive algebras: 
(P) If $\mathbb{Q}$ is any reductive algebra such that $\mathbb{Q}^{\prime}$ contains $T$, then $\mathbb{Q}^{\prime}$ contains $T^{*}$.

Rosenthal then asked if $T$ has property (P) under each of the following conditions:

(1) $T$ is polynomially compact,

(2) $1-T^{*} T$ is in some $C_{p}$ class,

(3) $T^{*}-T$ is in some $C_{p}$ class,

(4) $T$ is a part of a finite-multiplicity backward shift.

We will show that each of the above conditions implies (P), but we need a preliminary result (Lemma 2).

In [5], the following lemma is proved:

LEMMA 1. Let $C$ be a nonzero compact operator, and suppose that $B$ is an operator such that every subspace that reduces both $B$ and $C$ and has dimension greater than 1 properly contains a nonzero subspace that reduces $B$ and $C$. Then $B$ and $C$ have a common reducing eigenvector.

The argument used to prove this lemma, with minor (and obvious) modifications will yield the following fact:

Lemma 2. Let $B$ be an operator and $C$ a nonzero compact operator. Suppose that

(1) Every hyperinvariant subspace of $B$ reduces $B$ and $C$.

(2) Every hyperinvariant subspace of $B$ of dimension greater than 1 properly contains a nonzero hyperinvariant subspace of $B$.

Then $B$ and $C$ have a common reducing eigenvector.

Proof. See [5, p. 230].

We are now ready to answer Rosenthal's questions. It should be remarked that C. K. Fong [2] has proved part (1) of the following theorem.

THEOREM 4. An operator T has property (P) under any one of the following conditions:

(1) $T$ is polynomially compact,

(2) $T^{*}-T$ is in some $C_{p}$ class,

(3) $1-T^{*} T$ is in some $C_{p}$ class,

(4) $T$ is a part of some finite-multiplicity backward shift.

Proof. (4) follows from (3), because the multiplicity of the shift of which $T$ is a part is the rank of $\sqrt{1-T^{*} T}$. (See [3, p. 278].) If this rank is finite then so is the rank of $1-T^{*} T$.

Next we remark that each of the conditions (1), (2), (3) is inherited by direct summands, and that each condition guarantees the existence of hyperinvariant subspaces [8, Corollaries 6.13, 6.15, 6.16] and [7, Theorem 1.1].

We will prove in detail that (2) implies that (P) holds; the proofs for (1) and (3) are analogous. Let $C=T^{*}-T$ and suppose that $C$ is in some $C_{p}$ class. We also suppose that $Q$ is a reductive algebra and that $T \in \mathbb{Q}^{\prime}$. Let $\mathfrak{T}_{0}$ be 
the subspace of Theorem 2 ; note that $\mathscr{T}_{0}$ reduces $\mathcal{Q}, T$, and $C$, and that $\left(T^{*} \mid \Re_{0}\right) \in\left(\mathbb{Q} \mid \Re_{0}\right)^{\prime}$. Thus it suffices to consider the case where $T$ is hyporeductive (by the remark following the Corollary to Theorem 3).

Since $T$ is hyporeductive, the space Th spanned by all the eigenvectors of $T$ reduces $Q, T$, and $C$, and $\left(T^{*} \mid \Re_{1}\right) \in\left(\mathbb{Q} \mid \Re_{1}\right)^{\prime}$ (the last statement follows by Lemma 5 of [1]); thus it suffices to consider the restriction of $Q, T$, and $C$ to $\mathfrak{R}_{1}^{\perp}$; i.e., we consider the case where $T$ has no eigenvalues and is hyporeductive.

After these reductions suppose $C$ is nonzero. Because $T$ is hyporeductive, every hyperinvariant subspace of $T$ reduces $T$ and $C$. Further, suppose $\mathscr{T}$ is a hyperinvariant subspace of $T$, of dimension greater than 1 . Then $\mathfrak{N}$ reduces $T$ and $C$, and $\left(T \mid \Re()^{*}-(T \mid \Re)\right.$ lies in some $C_{p}$ class. Thus there is a hyperinvariant subspace of $T$ properly contained in $\mathfrak{T}$. It now follows from the assumption that $C$ is nonzero, and from Lemma 2 , that $T$ has a reducing eigenvector; however, we reduced to the case where $T$ has no eigenvectors. Thus it must be that $C=0$, which means that $T^{*}=T$ and $T^{*} \in \mathbb{Q}^{\prime}$. The proof is complete.

To show that (1) implies (P) let $p$ be a polynomial such that $p(T)=C$ is compact and proceed as above. It is necessary to know that an algebraic hyporeductive operator is normal [1, Theorem 4].

To show that (3) implies (P) let $C=1-T^{*} T$ and proceed as above.

We remark that the proof of Theorem 4 also establishes the following fact :

COROLlaRY. If $T$ is hyporeductive and any one of conditions (1) through (4) holds, then $T$ is normal.

\section{REFERENCES}

1. C. K. Fong, On operators with reducing hyperinvariant subspaces (preprint).

2. __ On commutants of reductive algebras, Proc. Amer. Math. Soc. 63 (1977), 111-114.

3. P. R. Halmos, A Hilbert space problem book, Van Nostrand, Princeton, N.J., 1967.

4. T. B. Hoover, Operator algebras with reducing invariant subspaces, Pacific J. Math. 4 (1973), 173-179.

5. R. L. Moore, Reductive operators that commute with a compact operator, Michigan Math. J. 22 (1975), 229-233.

6. __ Hyperinvariant subspaces of reductive operators, Proc. Amer. Math. Soc. 63 (1977), 91-94.

7. C. Pearcy, J. R. Ringrose and N. Salinas, Remarks on the invariant subspace problem, Michigan Math. J. 21 (1974), 163-166.

8. H. Radjavi and P. Rosenthal, Invariant subspaces, Springer-Verlag, Berlin and New York, 1973.

9. P. Rosenthal, On commutants of reductive operator algebras, Duke Math. J. 41 (1974), 829-834.

Department of Mathematics, Bucknell University, Lewisburg, Pennsylvania 17837 